\title{
Representationalism, perceptual distortion, and the limits of phenomenal concepts
}

\author{
Forthcoming in Canadian Journal of Philosophy \\ David Bourget \\ University of Western Ontario
}

November 4, 2014

\begin{abstract}
This paper replies to objections from perceptual distortion (blur, perspective, double vision, etc.) against the representationalist thesis that the phenomenal characters of experiences supervene on their intentional contents. It has been argued that some pairs of distorted and undistorted experiences share contents without sharing phenomenal characters, which is incompatible with the supervenience thesis. In reply, I suggest that such cases are not counterexamples to the representationalist thesis because the contents of distorted experiences are always impoverished in some way compared to those of normal experiences. This can be shown by considering limit cases of perceptual distortion, for example, maximally blurry experiences, which
\end{abstract}


manifestly lack details present in clear experiences. I argue that since there is no reasonable way to draw the line between distorted experiences that have degraded content and distorted experiences that don't, we should allow that an increase in distortion is always accompanied by a degradation in content. I also discuss the prospects for a positive account of the contents specific to distorted experiences. I argue that the prospects for such an account are dim, but that this is due to limitations of our phenomenal concepts, not to the falsity of the representationalist thesis.

\section{Introduction}

One of the central tenets of representationalism about consciousness is that the phenomenal characters of sensory experiences supervene on their intentional contents (perhaps in conjunction with their sensory modalities). Many of the putative counterexamples to this thesis involve perceptual distortion, for example, blur, double vision, perspective, and lighting conditions. ${ }^{1}$ In the case of blur, the problem for the representationalist is that there seem to be pairs of blurry and non-blurry experiences that have the same content but differ in phenomenal character. ${ }^{2}$ Double vision, perspective, and lighting

Many thanks to Angela Mendelovici for extensive feedback and very helpful suggestions. Thanks also to David Chalmers, Matthew Fulkerson, and Robert Howell.

${ }^{1}$ I count as perceptual distortion any normal variation in phenomenal character that is not normally associated with how things are in the environment independently of the perceiver and her relation to the environment.

${ }^{2}$ See for example Boghossian and Velleman (1989), Kuczynski (2004), and Smith (2008). 
conditions also seem to generate counterexamples of this kind. The general threat to representationalism is that perceptual distortion seems to generate distortion counterexamples to the supervenience thesis, where a distortion counterexample would be a pair of experiences that differ with respect to one type of perceptual distortion or another and, as a result of this, exhibit different phenomenal characters while having the same intentional content.

This paper offers a general argument against the existence of distortion counterexamples. Here is an outline of the argument: 1) A large amount of perceptual distortion is invariably accompanied by a significant loss of content. For example, a highly blurry experience cannot represent the same details as a normal experience. 2) If high levels of distortion invariably involve a significant loss of content, it is plausible that any level of distortion involves some loss of content, because there seems to be no principled way of drawing the line between lossy and non-lossy distortion. Therefore, it is likely that experiences that differ only with respect to the amount of distortion they exhibit do not constitute counterexamples to representationalism. I will refer to this as the amplification argument. My main goal in this paper is to develop the amplification argument.

Even if one agrees that the amplification argument shows that there are no distortion counterexamples, one might think that representationalism can only be made plausible by providing an account of the manner in which the phenomenal characters of distorted experiences are determined by their contents. Several accounts have been offered, but none seems to have gained 
widespread acceptance, even among committed representationalists. ${ }^{3}$ This might seem to speak against the overall representationalist framework. A secondary aim of this paper is to make the case that these difficulties do not speak against representationalism. I will argue that the reason we have difficulty accounting for the phenomenal characters of distorted experiences in terms of their contents is that we lack sufficiently articulate phenomenal concepts for these phenomenal characters, not that the supervenience thesis is false.

Some terminological clarifications: An experience is an instantiation of a phenomenal state, where a phenomenal state is a state individuated by its phenomenal character (what it is like to be in it). For the purposes of this paper, I equate representationalism with the claim that the phenomenal characters of experiences supervene on their intentional contents. ${ }^{4}$ I will leave open how we should understand the notion of intentional content that figures in the representationalist thesis. There are several acceptable ways of fixing the reference of "intentional content." Each way yields a different representationalist thesis. Personally, I am primarily interested in the thesis we obtain when we take intentionality to be the kind of directedness that accounts for subjects' points of view. Accounting for subjects' points of view

\footnotetext{
${ }^{3}$ See for example Schroer (2002); Tye (2003); Dretske (2003); Kuczynski (2004); Pace (2007); Allen (2013).

${ }^{4}$ Some representationalists hold the weaker view that phenomenal character supervenes on content together with sensory modalities, but this view is also incompatible with the kinds of putative counterexamples discussed here. For more on representationalism, see Lycan Lycan (2000), Seager \& Bourget (2007), and Bourget \& Mendelovici (2014)
} 
is a matter of accounting for how things seem to them, how things strike them, how they think, and what motivates them. So I am inclined to take intentionality to be the central feature of the states we are in when certain things seem true or desirable to us, and I am inclined to take intentional contents to be the things that states involving this feature are directed at. ${ }^{5}$ Other readings of the representationalist thesis are also worth considering. My argument in this paper is largely neutral on these matters. The reader who is more comfortable with another definition of intentional content (say, in terms of satisfaction conditions or tracking) is welcome to understand content talk in this way while keeping in mind that the argument is primarily intended to defend the representationalist thesis on the reading just specified (minor tweaks may be required to adapt the argument to alternate notions of content).

\section{The case of blur}

I will begin by developing my response to the argument from perceptual distortion for the case of blur.

\footnotetext{
${ }^{5}$ This definition of intentionality is only meant to fix reference in a partially ostensive manner, not to spell out what intentionality is. In this regard, I am following the example of Mendelovici (2010) and Kriegel (2011). Elsewhere (2010a, 2010b), I've favoured another definition of intentionality according to which it consists in standing in a certain kind of fallible relation to a proposition-like object. I believe that these definitions are coextensive, but it is non-trivial to show this. Interesting questions can be formulated in terms of either definition.
} 


\subsection{The objection}

The objection from blur was introduced into the contemporary literature by Boghossian and Velleman:

... by unfocusing your eyes, you can see objects blurrily without being able to see them as being blurry. None of these experiences can be adequately described solely in terms of their intentional content. (Boghossian \& Velleman, 1989, p. 94)

I will say a few words about this version of the objection before moving on to what I take to be the main objection from blur.

Boghossian and Velleman are naturally interpreted as making an argument along the following lines (other readings that I am not going to discuss here are possible, but they are not very charitable):

1. Blurry visual experiences do not represent things as blurry.

2. If phenomenal character supervenes on content, then blurry visual experiences represent things as blurry.

3. Therefore, phenomenal character does not supervene on content.

One problem with this argument is that representationalists have good reasons to deny premise 2. Boghossian and Velleman do not defend premise 2 in any way. It is largely tacit in their argumentation; they seem to think that it unpacks an obvious consequence of representationalism. Presumably, they 
think that the following generalization of premise 2 also states a consequence of representationalism:

Insertion If phenomenal character supervenes on content, then, for any property $F$, every experience that has $F$ represents something as $F$. Insertion does not state a necessary consequence of representationalism. On the contrary, those who endorse its antecedent claim (the representationalist supervenience thesis) should reject it. Several examples show this. For instance, a representationalist should agree that late morning experiences do not normally represent anything as (occurring in) the late morning, but this should not make her doubt her view that the phenomenal characters of these experiences supervene on their contents. Drunken perceptual experiences do not normally represent anything as drunk, but this does not mean that the phenomenal characters of these experiences do not supervene on their contents. Every representationalist should agree that many properties falsify Insertion. As a result, a natural response for the representationalist to make to Boghossian and Velleman's argument is to deny premise 2.

Of course, one might reply that, while many properties falsify Insertion, some confirm it, and it is possible that blurriness falls in the latter category. To shed further light on the status of premise 2, we need a way of distinguishing those properties that do and do not satisfy Insertion. If we knew the appropriate criterion, we would be able to tell whether premise 2 is true or not. The preceding examples suggest the following partial criterion: if $F$ is a property of experience that is not fully determined by the phenomenal 
character of its bearer (e.g. occurring in the late morning, or being generated by a drunk brain), then $F$ does not satisfy Insertion. Put a little more loosely, it seems plausible that representationalists' supervenience claim commits them at best to the view that the properties of experiences that pertain exclusively to their phenomenal characters figure in their contents, not to the claim that all properties of experiences figure in their contents. This means that, unless blurriness pertains exclusively to phenomenal character, the representationalist is entitled to deny premise 2 .

Now, a moment's reflection suggests that blurriness does not pertain exclusively to phenomenal character. When the projection is out of focus at the cinema, one's visual experiences are not blurry in the usual sense, but they have phenomenal characters very close to those of typical blurry experiences. It is not hard to imagine their having phenomenal characters identical to those of blurry experiences. This shows that for an experience to count as blurry, it is not enough that it have a certain kind of phenomenal character (hence blurriness does not pertain exclusively to phenomenal character). ${ }^{6}$ The same holds of blurry photographs: if you take a photo of an out-of-focus projection, you might end up with a clear picture that is qualitatively like a blurry picture. To count as blurry, an experience or a photograph must be the product of a certain kind of (defective) optical or imaging process that distorts it in a certain way compared to the norm. So blurriness is a property that goes beyond phenomenal character. In this regard, being a

\footnotetext{
${ }^{6}$ See Seager \& Bourget (2007) for another discussion of this kind of case.
} 
blurry experience is like being a drunken experience. For this reason, it is perfectly reasonable to maintain that phenomenal character supervenes on content even though blurriness never figures in the content of any experience.

Of course, there is a closely related property that pertains to phenomenal character. In this world at least, blurry experiences all have a certain kind of "fuzzy" phenomenal character. Call this type of phenomenal character blurriness' ${ }^{7}$ One might think that Boghossian and Velleman's argument is sound if we read "blurry" as meaning blurry'. Blurriness' at least avoids the concerns that we raised with blurriness.

There is still a problem with premise 2 on this reading. Even if we restrict $F$ to properties that pertain exclusively to phenomenal character, such as blurriness', Insertion still has many false instances. Take the property of having a simple phenomenal character. This property pertains exclusively to phenomenal character, but it is clear that it need not figure in the contents of experiences for representationalism to be true. All properties that merely characterize the overall flavour of a phenomenal character seem to make exception to Insertion. One might reasonably think that blurriness' falls in this category.

\footnotetext{
${ }^{7}$ I fix reference on blurriness' through actual blurry experiences because I don't think that blur is necessarily associated with any sort of phenomenal character. It seems to me that there could be situations where blurry experiences (that is, experiences that are distorted in the same kind of way as blurry experiences in this world) have phenomenal characters identical to those of clear experiences in this world. I can imagine the sort of distortion that is characteristic of blur rearranging a "fuzzy input" to create a "clear image." It also seems possible for a clear experience to have a phenomenal character identical to that of a blurry experience in the actual world, as mentioned above.
} 
These remarks are not fatal to the argument from blur, but they help focus on the real point of contention. Boghossian and Velleman seem to construe representationalism in such a way that it commits us to the view that all the properties of an experience figure in its content. This construal of representationalism is captured by the Insertion claim. Insertion goes beyond the supervenience claim that is the core representationalist thesis, and representationalists arguably have independent reasons for rejecting Insertion. So if blur makes trouble for representationalism on the assumption that Insertion or a similar claim is true, it is natural for a representationalist to reject Insertion and all similar claims. A better argument would make blur directly relevant to the representationalist supervenience claim without additional assumptions along the lines of Insertion. Such an argument would have to go as follows:

1. Some blurry and non-blurry (or less blurry) experiences have the same content but distinct phenomenal characters.

2. If (1), phenomenal character does not supervene on content.

3. Therefore, phenomenal character does not supervene on content.

In my view, this argument best captures the core objection from blur. Here premise 2 is analytic, so all the weight of the argument rests on premise 1.

Premise 1 is much less obvious than Boghossian and Velleman's original starting point (the correct but, as I have argued, irrelevant observation that blurry experiences do not represent anything as blurry). Premise 1 
simply states that blur generates distortion counterexamples. Boghossian and Velleman, like many others who have thought about this topic, seem to think that this claim is justified on the basis of phenomenological observation in familiar cases of blur. The key question in this debate is whether phenomenological observation really does support premise 1, the claim that blur generates distortion counterexamples. In the next subsection, I will argue that phenomenological observation militates against premise 1, not for it.

\subsection{The amplification argument}

I must stress that the question I want to address is how we should describe ordinary blurry experiences. I take it that the objections from perceptual distortion draw their force in good part from the fact that they are based on phenomenological observations that everyone is supposed to be able to validate at home (as opposed to mere thought experiments). If it can be shown that ordinary cases do not generate distortion counterexamples, this type of argument will be severely undermined. Put a little more formally, the question is whether distortion counterexamples are psychologically possible, where psychological possibility is possibility given the way our minds and brains work. I will briefly return to the metaphysical possibility of distortion counterexamples in section 4 , but we cannot hope to settle this issue here, because it raises a host of difficult questions regarding the epistemology of modality. 
Consider Figure 1. Let us suppose that $S_{0}$ illustrates the phenomenal
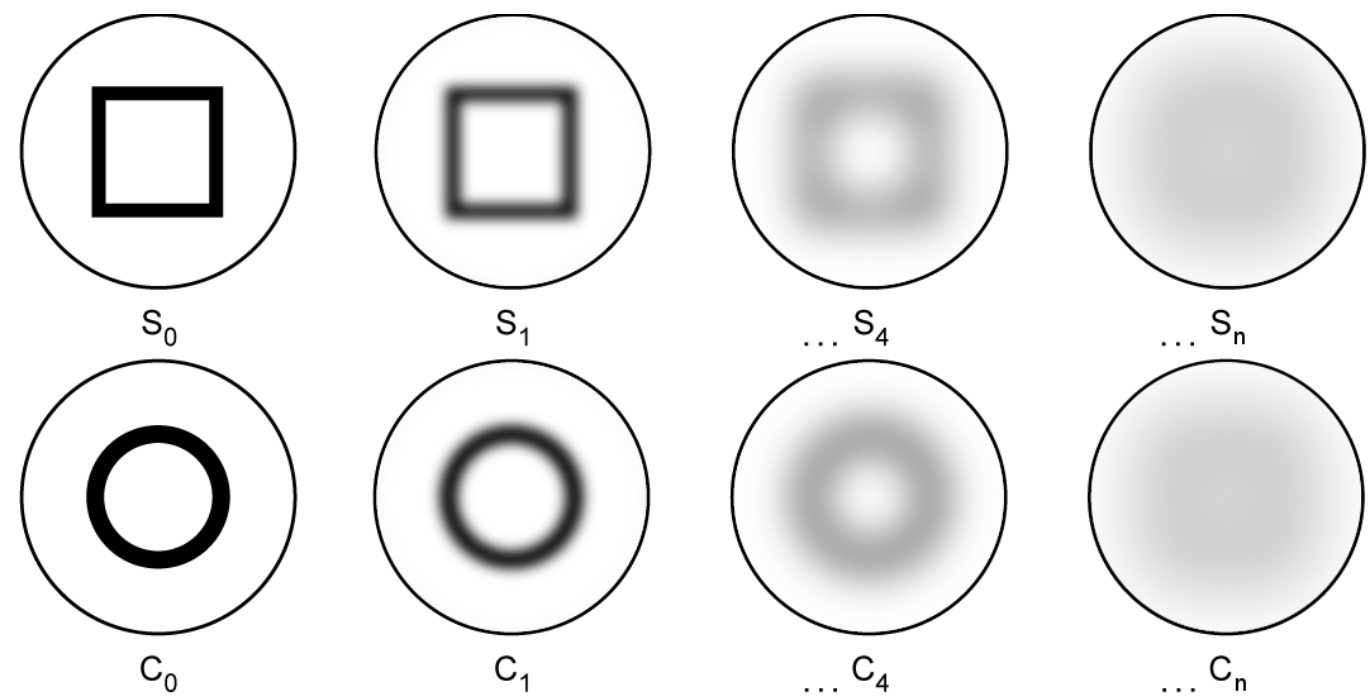

Figure 1: Series of increasingly blurry experiences

character of a maximally clear experience caused by the sight of a square shape. $\mathrm{S}_{1} \ldots \mathrm{S}_{n}$ depict the phenomenal characters of experiences that one would normally have if one looked at the same shape while one's vision is affected by different levels of blur (from the smallest to the largest amount of blur). Looking at $\mathrm{S}_{0}$ and $\mathrm{S}_{1}$, one might think that these experiences have the same content, namely the content there is a square in front of me, or some such. This appears to be the position of proponents of the objection from blur.

That there is a difference in content between $S_{0}$ and $S_{1}$ can be made clear by amplifying the effect of blur. It seems undeniable that $\mathrm{S}_{0}$ and $\mathrm{S}_{n}$ differ in content. In case one is tempted to deny this, consider that $S_{n}$ is 
phenomenally identical to $\mathrm{C}_{n}$, another extremely blurry experience, but one that is the limit case of blurry vision of a circle. $\mathrm{S}_{n}$ and $\mathrm{C}_{n}$ must have the same content on any reasonable understanding of the notion of content. If content accounts for subjects' point of view, for example, they must have the same content because they yield the same point of view. If we allowed that $\mathrm{S}_{n}$ has the same content as $\mathrm{S}_{0}$, we would have to allow that $\mathrm{C}_{n}$ has the same content as $\mathrm{C}_{0}$. But given the identity of $\mathrm{C}_{n}$ 's and $\mathrm{S}_{n}$ 's contents, this would have the absurd consequence that $\mathrm{C}_{0}$ and $\mathrm{S}_{0}$ have the same content. Clearly, things seem different in $\mathrm{C}_{0}$ and $\mathrm{S}_{0}$.

Given that there is a change in content between $\mathrm{S}_{0}$ and $\mathrm{S}_{n}$, the question is where we should locate it along the series. There are three options:

1. A big shift: there is an $i$ between 1 and $n$ such that $\mathrm{S}_{0} \ldots \mathrm{S}_{i-1}$ have the content of $S_{0}$ and $S_{i} \ldots S_{n}$ have the content of $S_{n}$, which is distinct from the content of $\mathrm{S}_{0}$.

2. Multiple shifts: multiple shifts in content occur between $\mathrm{S}_{0}$ and $\mathrm{S}_{n}$, but some consecutive experiences in series $\mathrm{S}$ have the same content.

3. Continuous change: every experience in $\mathrm{S}$ differs in content from its predecessor.

A big shift seems highly implausible. For one thing, where should we draw the line (what should we take $i$ to be)? There does not seem to be any non-arbitrary way to select $i$. One might suggest that the shift occurs at the point where one stops recognizing a square shape, but the information that 
a square shape is present is just one element of the content of $\mathrm{S}_{0}$. That is not all that is represented in $\mathrm{S}_{0}$ but not $\mathrm{S}_{n}$. Other features that figure in the content of $\mathrm{S}_{0}$ but not that of $\mathrm{S}_{n}$ include:

- A squarish shape

- Eight right angles

- Four line-like shapes

- The sharpness of the four sides

- The length and width of the four sides

- The shade of every discernible point of the square figure

- The shade of every discernible point of the area within the figure

All of the above are features that a subject of $S_{0}$ is sensitive to: they make a potential difference, however small, to how the world seems to the subject at the time of having the experience. So they are plausibly part of the content of $S_{0}$ in some way. It is possible that some are part of the content of $S_{0}$ in a way that makes it hard for us to isolate them, but the content of $\mathrm{S}_{0}$ must capture them in some way or other, because we can potentially recognize them based on $\mathrm{S}_{0}$ alone (given the relevant concepts).

Given that all of the above features are represented by $\mathrm{S}_{0}$ but not $\mathrm{S}_{n}$, it seems implausible that there is just one big shift in content between $\mathrm{S}_{0}$ and $\mathrm{S}_{n}$. In particular, it seems clear that one can continue to remain aware of 
squarishness or line-like shapes after one has lost the information that the shape is perfectly square or has such and such exact contours. The features listed above do not all vanish at the same time from the content of one's experience.

A multitude of shifts (option 2) is more plausible. One might think that the features listed above disappear from the content of one's experience at different points along series S, yet that some changes in phenomenal character along series S do not correspond to changes in content.

Aside from its ad hoc character, one difficulty with this hypothesis is that the above list seems to be indefinitely extensible. In principle, all sub-areas of $\mathrm{S}_{0}$ and all their abstract properties are features that one can notice as part of $\mathrm{S}_{0}$. An infinite number of discontinuous shifts seems implausible, but it is hard to see how one might go about attributing each of these features to a limited number of shifts. Another problem is that certain non-categorical features we are aware of in experiences like $\mathrm{S}_{0}$ seem to be lost progressively. Take for example the area of the figure. Every small amount of blur added removes information about the area of the figure without there being at any point a shift from representing the area to not representing it at all. Every small amount of blur added also subtracts some information about the colours of certain minimally discernible surfaces of the figure: one becomes increasingly unclear on their colours. The same holds of many other noncategorical features of the figure, for example, the sharpness of the corners or the length of the sides. Blur progressively takes away information about 
these features. The account of series $\mathrm{S}$ that is most plausible in light of these observations is that series $\mathrm{S}$ experiences lose content at every step (option 3). Therefore, no pair of consecutive experiences in series $\mathrm{S}$ is a distortion counterexamples to the representationalist supervenience thesis. Assuming that contents do not repeat in series $\mathrm{S}$, it follows that no pair of experiences in series $\mathrm{S}$ is a distortion counterexample.

There is nothing special about series $\mathrm{S}$, and any two experiences that differ only in the level of blurriness they exhibit can be part of a series such as S. Consequently, it is natural to generalize the previous reasoning to all (psychologically possible) cases of blur. It seems plausible that no pair of experiences that differ only with respect to their level of blurriness is a distortion counterexample to the representationalist thesis.

Note that the claim that blur causes a loss of content is not equivalent to Tye's (2003) account of blur. Tye suggests that the difference between blurry experiences and normal experiences lies in the fact that the former have indeterminate contents. While indeterminacy is one possible explanation for the loss of content brought out by the amplification argument, it is not the only possible explanation. There are other ways for $S_{i}$ to represent features that $S_{i+1}$ does not represent than for $S_{i+1}$ to have indeterminate content. It could simply be that $S_{i}$ represents aspects of the scene that $S_{i+1}$ does not represent (in the same way that the belief that $P \wedge Q$ represents more than the belief that $P$, provided that $P$ does not entail $Q$ ). It could also be that while blurry experiences fail to represent certain features that non-blurry 
experiences represent, they represent other things instead, for example, fuzzy properties. For now I am remaining neutral on these questions. I will say more about the contents of distorted experiences in section 5 .

\section{Other forms of perceptual distortion}

The amplification reply generalizes to other arguments from perceptual distortion. Consider the effect of distance on phenomenal character and representational content, another kind of perceptual distortion that has been thought to generate counterexamples to representationalism. Take Peacocke's example of the two identically sized trees seen from different distances:

Suppose you are standing on a road which stretches from you in a straight line to the horizon. There are two trees at the roadside, one a hundred yards from you, the other two hundred. Your experience represents these objects as being of the same physical height and other dimensions; that is, taking your experience at face value you would judge that the trees are roughly the same physical size ... Yet there is also some sense in which the nearer tree occupies more of your visual field than the more distant tree. (Peacocke 1983, p. 12)

Although this was not exactly Peacocke's intent here, this case has often been taken to challenge representationalists' claim that no two experiences 
can differ in phenomenal character without differing in content: it seems that one's experiences of the two trees on the road could have the same content even if they had different phenomenal characters corresponding to the different apparent sizes of the trees. ${ }^{8}$ This case seems to show that distance can distort phenomenal character without affecting content.

Against this, consider a similar case illustrated by Figure 2. You are

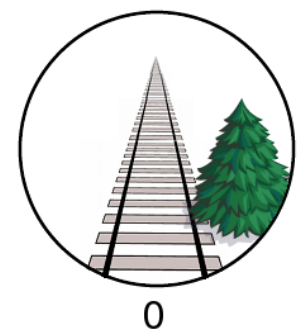

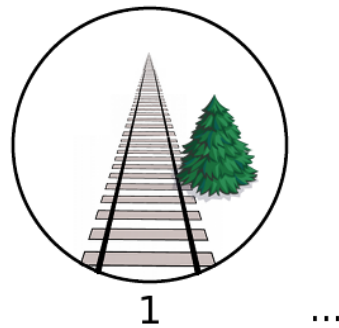

1

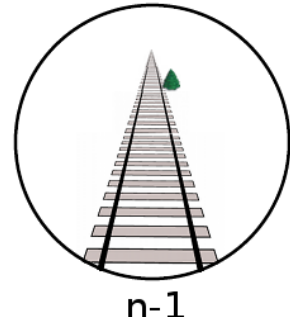

n-1

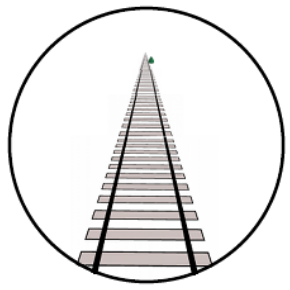

$\mathrm{n}$

Figure 2: The effect of distance (series T)

standing at the back of a train that has just passed by a pine tree. As the train moves away from the tree, you go through a series of tree experiences $\mathrm{T}_{0}$ to $\mathrm{T}_{n}$ (we abstract way from the rest of the scene). It might seem at first that there is no change in the content of your experience as the train moves away. After all, you do not think that the tree is shrinking. But now consider the limit case. Many features of the tree that are represented in $\mathrm{T}_{0}$ are not

\footnotetext{
${ }^{8}$ Millar (2010) and an anonymous referee point out that Peacocke explicitly denies trying to refute the representationalist supervenience claim with this example (see Peacocke 1983, p. 13). But the example has nevertheless often been interpreted in this way, as Millar notes (see for example Lycan (1996), Tye (1996) and Chalmers (2004)). Setting aside the exegetical question, this is a representative example of the type of distance-based argument from perceptual distortion against representationalism one encounters in print and in conversation.
} 
represented in $\mathrm{T}_{n}$ : the individual branches, the colours of their multiple surfaces, the precise jagged contours of the branches, the overall shape of the tree, etc. As before, there are three possible hypotheses regarding what is happening to the contents of your experiences as you move farther away from the tree: a big shift, multiple shifts, or continuous change. As before, a big shift is implausible because different features are plausibly lost at different points. A multitude of shifts seems implausible because information about some non-categorical features seems to be lost progressively, for example, information about how conical the tree is. It is also possible to multiply the relevant features indefinitely. So the most plausible hypothesis is that the contents of experiences $T_{0} \ldots T_{n}$ change along with their phenomenal characters.

The amplification argument also applies to the case of viewing angle. Consider a series $\mathrm{A}_{0} \ldots \mathrm{A}_{n}$ of experiences obtained by moving around an object as illustrated by Figure 3. One might have thought that the change
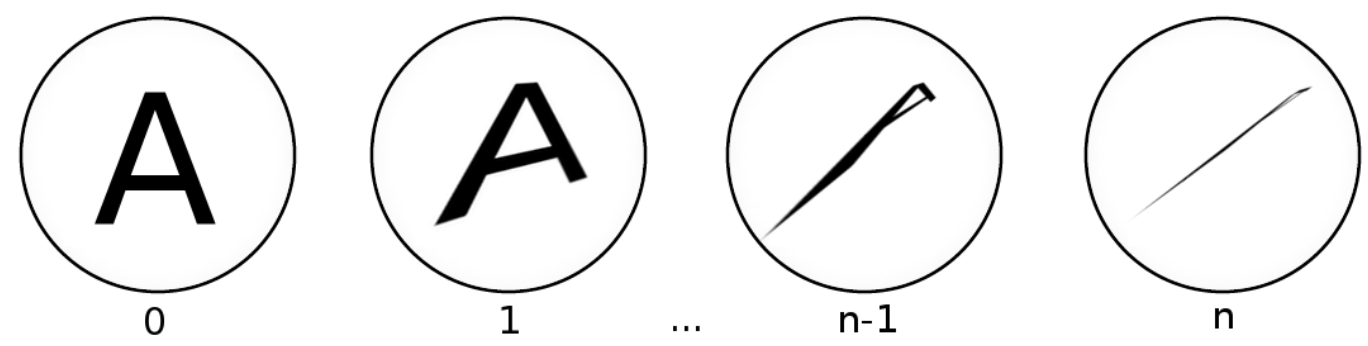

Figure 3: The case of viewing angle (series A)

from $A_{0}$ to $A_{1}$ does not correspond to a change in content, but that it does is 
evident when we amplify the change in question. In this case, it seems more plausible that a big shift occurs because many of the features are plausibly lost between $A_{n-1}$ and $A_{n}$. Still, other features are lost before $A_{n-1}$. For example, $\mathrm{A}_{n-1}$ contains less information about the texture and colour of the right leg of the "A" than $\mathrm{A}_{0}$ does: as this part of the object is moved further away, we can only lose some information about its surface. Since this information seems to be lost progressively, the best hypothesis is again that content changes continuously with phenomenal character.

Figure 4 illustrates experiences exhibiting increasing levels of double vision. ${ }^{9} \mathrm{D}_{o}$ exhibits no double vision and $\mathrm{D}_{n}$ exhibits extreme double vision. Double vision typically involves some amount of blur. Insofar as this is the
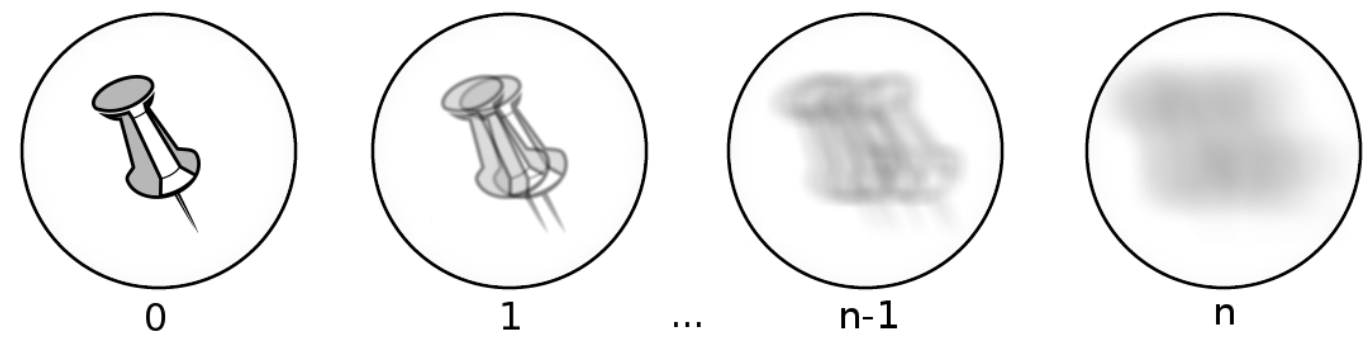

Figure 4: The case of double vision (series D)

\footnotetext{
${ }^{9}$ One might say that the limit case of double vision is not " $n$ vision" as illustrated in $\mathrm{D}_{n}$ and $\mathrm{E}_{n}$ but an instance of double vision where the two images seen are very far apart. The fact is that double vision involves more than one kind of distortion. Among other effects, there is a multiplication and superposition of images, and there is also a separation of images. For my purposes, it is sufficient that one aspect of double vision causes a loss of content. Here I am focusing on the multiplication and superposition aspect. My point is that amplifying this effect shows that it always involves a loss of content. This is sufficient to show that ordinary double vision, which always involves this effect, always involves a loss of content.
} 
case, the amplification argument should work just as well against double vision as it does against blur. Indeed, series D illustrates the fact that an extreme amount of double vision, which involves an extreme amount of blur, eclipses almost all useful information from visual experience. As before, this leaves open three possibilities: a big shift, multiple shifts, and continuous change. The hypothesis of continuous change seems by far the most plausible for the same reason as in series S: there is no plausible place to locate a big shift in the series, and some information seems to be lost continuously.

One might think that it is possible to have double vision without blur, at least in principle. Our concern here is only with psychologically possible experiences, but it does not hurt to consider the case. Experiences exhibiting double vision without blur would presumably look something like those in series E (Figure 5). This series illustrates the sort of superposition effect that one experiences (along with blur) in a normal double vision experience. This effect might seem not to make a difference in content in cases such
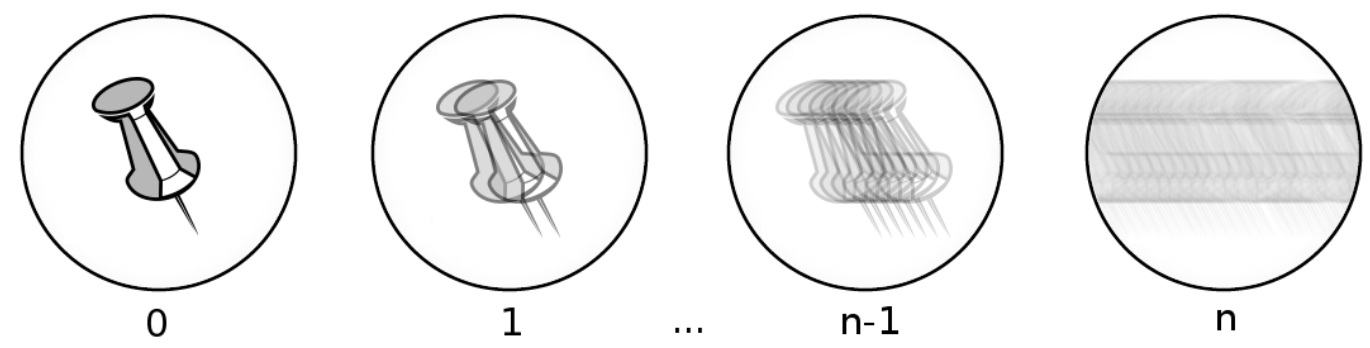

Figure 5: The case of double vision without blur (series E)

as $\mathrm{E}_{1}$, but that it does becomes clear when we amplify it, as in $\mathrm{E}_{n}$. In 
$\mathrm{E}_{n}$, much of the information that is present in $\mathrm{E}_{0}$ has been lost. By far the most plausible explanation is that the superposition effect that is part of double vision always takes away some content. Metaphorically speaking, double vision seems to "blend" multiple "images" of an object. There is an inevitable information loss whenever such an effect is applied.

We can also apply the amplification argument to the case of illumination conditions, which also seem to generate difficulties for the representationalist supervenience claim, at least on some views of the representational contents of experience. ${ }^{10}$ We generally take the colours of objects to be constant in spite of multiple variations in how they appear to us due to variations in lighting conditions (e.g. shadows). At first, it might seem that such changes affect the phenomenal characters of our experiences without affecting their contents. But shadows and other changes in illumination conditions that affect phenomenal character do affect how our experiences represent the world. ${ }^{11}$ In particular, they affect the details that are captured by the contents of our experiences. We can show this by amplifying the relevant illumination effects.

Take the case of shadows. Suppose that you are staring at the floor of your office, which is illuminated in sunlight. As you are staring at the floor,

\footnotetext{
${ }^{10}$ See for example Thompson (2006), Jagnow (2009), and Millar (2013).

${ }^{11}$ There seems to be two different kinds of colour constancy effects. Sometimes, phenomenal character seems to remain constant despite changes in illumination conditions. Other times, phenomenal character changes but our spontaneous judgments regarding colours do not. The first kind of case does not threaten representationalism, so it is the latter kind of case that is of interest to us here.
} 
a bird flies by the window, briefly blocking part of the light from the sun. The bird throws a faint shadow on the floor, and your experience of this part of the floor changes in phenomenal character. For lack of a better way of putting it, let us say that your experience acquires a darker phenomenal character. It might seem that this darkening in phenomenal character is not accompanied by a darkening in representational content. One might take the fact that you would not judge that the relevant part of the floor has changed as evidence that the representational content of your experience remains constant. While this might seem plausible at first, consider what happens if we intensify the perceptual distortion in question. We can imagine a series of cases like this one were the shadow is increasingly dark. In the limit case, the bird could block absolutely all light from reaching a certain bird-shaped area of the floor. In this case we have to say that your experience differs in content from the shadowless experience. For while the shadowless experience tells you about minute cracks, stains, and variations in texture in the floor within the shadowed area, the experience in the limit case tells you none of this. Because the information loss is progressive, it is reasonable to conclude that a darkening in illumination always makes a difference in content. As in other cases we discussed, to say that information is lost is not to say that a smaller amount of information is represented, because what is lost could be replaced. Perhaps your experience of complete darkness provides you with a lot of information of some kind. The point is only that some information found in the initial experience has been lost. 
Parallel arguments can be made regarding other kinds of variations in illumination conditions. Take for example the difference between early morning and noon light, or between overcast and clear conditions. These different illumination conditions generate different spectral power distributions (SPDs). Slightly different SPDs result in slightly different experiences that might seem to have the same representational contents, but large differences in SPD make obvious differences in content. If your environment is only illuminated in blue light, for example, your visual experiences will fail to reveal many features of your environment that they reveal in normal sunlight. Just like with shadows and other perceptual distortions, it is reasonable to suppose that a small difference in phenomenal character caused by a small difference in SPD always comes with a difference in representational content, because we can construct series of visual experiences involving progressive changes in SPD that involve a progressive loss of information about your environment. ${ }^{12}$

I take the preceding discussion to show that the amplification reply undermines the objections from blur, distance, viewing angle, double vision, and lighting conditions against representationalism. I also take this discussion to suggest that other kinds of perceptual distortion not explicitly discussed here (for example, the sort of distortion that affects peripheral vision) can likely be addressed in a similar way.

\footnotetext{
${ }^{12}$ Of course, this does not commit us to the view that any difference in SPD makes a difference to the contents of visual experience: only differences that affect phenomenal character make such a difference.
} 


\section{Are distortion counterexamples metaphys- ically possible?}

As I noted earlier, the amplification argument is primarily intended to bear on psychologically possible experience pairs, not all metaphysically possible experience pairs. Still, it can provide some evidence against the metaphysical possibility of distortion counterexamples, given certain assumptions regarding the epistemology of modality. I will explain how the amplification argument is relevant to metaphysically possible cases, but I will not attempt to defend the required assumptions, because this would take us too far afield.

There seem to be two possible ways of a priori assessing whether or not a scenario that is not actual is metaphysically possible. The first way is through formal proof, a method that is clearly not applicable to distortion cases. The second way is through imagination or pure rational intuition. On this method, one tests a scenario $S$ for metaphysical possibility by attempting to imagine or intuit the possibility of a concrete situation in which $S$ is the case. If one can imagine or intuit such a situation, this provides some (albeit non-conclusive) evidence that $S$ is possible. Conversely, if one cannot imagine or intuit such a situation, this provides some (albeit far from conclusive) evidence that $S$ is impossible. I am going to call this the method of imagination. ${ }^{13}$ For ease of exposition, I will assume that the relevant kind of

\footnotetext{
${ }^{13}$ The method of imagination is my take on Chalmers' (2002) positive conceivability method.
} 
mental act can be described as imagining.

An important limitation on the applicability of the method of imagination is that one must have a sufficient conceptual grip on the nature of the situation whose possibility one is assessing. Otherwise, one's imagination or intuition will not be aiming at the right target (if anything at all). In the case of "Water is not $\mathrm{H}_{2} \mathrm{O}$," for example, one might fail to know that this statement expresses the necessarily false proposition $\neg\left(\mathrm{H}_{2} \mathrm{O}=\mathrm{H}_{2} \mathrm{O}\right)$. In this case, one would be unable to test the possibility of the scenario through imagination because one would not know what is to be imagined. In order to be in a position to use the method of imagination, one must have a sufficient conceptual grip on the scenario or proposition in question.

When the methods of proof and imagination are not applicable, we can only determine the possibility of counteractual scenarios on the basis of theories of the nature of things grounded in empirical evidence from the actual world. For example, our best theory of water tells us that water is $\mathrm{H}_{2} \mathrm{O}$, which rules out the possibility that water is not $\mathrm{H}_{2} \mathrm{O}$. This is a case that could only be adjudicated on the basis of empirical evidence.

Assuming that the preceding is correct, we might be in a position to say something about the metaphysical possibility of distortion cases simply by reflecting on the cases that we can imagine. Consider first the case of blur.

Here it is important to keep in mind the point made earlier that blur is not intrinsic to phenomenal character. Among the metaphysically possible worlds, there might well be worlds where some blurry experiences are phe- 
nomenally identical to clear experiences in the actual world, and there might be worlds where some clear experiences are phenomenally identical to blurry experiences in the actual world. What we are interested in here is blurriness', the phenomenal type that is characteristic of (normal) blurry experiences in the actual world. The question is whether a difference in blurriness ${ }^{\prime}$ always comes with a difference in content.

Every blurry' experience that I can imagine has a phenomenal character that exhibits the same kind of "blending of pixels" we see in $\mathrm{S}_{1}$. When asked to imagine a blurry' experience, I do not know what to picture unless it is an experience that is blended in this way. Moreover, if I imagine an experience that is more blurry' than another, the more blurry' experience is more blended than the less blurry' experience. Therefore, every blurry' experience I can imagine seems to lie on a possible series of increasingly blurry' experiences exhibiting increasingly blended pixels just like the experiences in series S. In all such imaginable series, extremely blended experiences clearly lack content that is present in unblended experiences. This difference in content needs to be accounted for. As in the case of series $\mathrm{S}$, there are three possible ways to account for it: a big shift, a multitude of shifts, and continuous change. Continuous change seems to be the most plausible hypothesis for exactly the same reason as in the case of series $\mathrm{S}$ : even in my imagination, the kind of blending that accompanies blur' seems to take away some aspects of the contents of my experiences progressively. So it seems reasonable to think that distortion counterexamples involving blur' cannot even be imagined. If 
the method of imagination is applicable to this case, this provides some evidence that distortion counterexamples involving blur' are not metaphysically possible.

The same is true of other kinds of perceptual distortion. In all cases, the experiences that I can imagine share with the examples above the features that seem to imply a loss of content. Insofar as I have a sufficiently good grip on the nature of the relevant experiences to use the method of imagination, the method seems to suggest that there are no possible distortion counterexamples.

At this point we are in a position to draw a tentative conclusion about the metaphysical possibility of distortion counterexamples without even settling the question of whether or not the method of imagination is applicable to this case (or to any case). If the method is applicable, its application combined with the amplification argument suggests that distortion counterexamples are not metaphysically possible, as we just saw. If the method is not applicable, then we have no way of assessing the mere metaphysical possibility of distortion counterexamples a priori. We can only justify judgments about such cases based on theories justified on the basis of actual cases and general theoretical considerations. In other words, all that we can do is treat merely possible cases as spoils for the victor. To the extent that the amplification argument can show that there are no actual distortion counterexamples, it also provides indirect support for the view that there are no counterfactual distortion counterexamples by making representationalism more plausible as 
a theory of the nature of experience. Whether or not the method of imagination is applicable to the cases at hand, the amplification argument provides some support for the impossibility of distortion counterexamples.

\section{The contents of distorted experiences}

So far I have argued that changes in phenomenal character due to perceptual distortion always involve a loss of content, and so that distorted and undistorted experiences always differ in content. Of course, not all forms of perceptual distortion affect content in exactly the same way. I do not claim that the only representational difference between distorted and undistorted experiences is that the latter lack contents that the former possess. Plau-

sibly, each kind of perceptual distortion transforms content in a distinctive way that involves more than subtraction. So far, I have remained neutral on what is going on representationally in perceptual distortion aside from the fact that distorted experiences lack contents that undistorted experiences have.

While the amplification reply can be appreciated without committing ourselves to any particular account of the specific variations in content that characterize each kind of perceptual distortion, one might think that a complete representationalist theory ought to specify exactly what are the specific contents of distorted experiences that account for their characteristic phenomenal characters. There are several proposals on the table, but none is 
widely accepted among representationalists. One might take these difficulties to indicate a general failing with the representationalist research program. I would like to conclude by addressing this concern.

I do not have complete accounts of the contents of distorted experiences to offer here. In fact, I believe that there is reason to think that compelling accounts are not forthcoming. The point I want to make is that we should not draw pessimistic conclusions about representationalism from these difficulties.

I will start by briefly reviewing existing accounts. In the interest of space, I will limit myself to the cases of blur and perspective. There are three main views regarding the contents of blurry experiences: Dretske (2003) suggests that they represent fuzzy properties; Tye (2003) holds that they have indeterminate contents, in the sense of contents which do not specify exactly how things are (where the boundaries of objects are, for example); Allen (2013) argues that blurry experiences represent objects as having multiple boundaries. All three proposals have some prima facie plausibility and echo the phenomenology of blurry experiences to some degree. All three proposals are also compatible with our observations from the previous sections.

There are two main proposals on the table concerning perspective. According to Lycan (1996), we need two layers of content to explain perspective: one that represents objects in objective three-dimensional space, and one that represents objects in an egocentric space. Lycan's response to Peacocke's example of the two tree experiences is that these two experiences share a 
layer of content (the objective one) while differing with respect to the other layer. The other proposal is Tye's (1996), who suggests that the difference in content between the two tree experiences is one in situation-dependent properties such as being large from here. ${ }^{14}$ Both Lycan's and Tye's proposals are compatible with our observation from the previous sections.

One might take the lack of convergence in accounts of the contents of distorted experiences to indicate a general failing with the representationalist research program. I will now explain why I think we should refrain from drawing pessimistic conclusions about representationalism on such grounds.

We must first get clearer on what we are trying to achieve. We would like an account of the specific contents of distorted experiences that explains their phenomenal characters. It would not be enough for a representationalist to say simply that the contents of distorted experiences are the contents that necessitate distorted phenomenal characters. What we are looking for is an intrinsic specification of the contents of distorted experiences that articulates the nature of these contents in such a way that one can see how they give rise to the relevant phenomenal characters. Part of what needs to be done here is to specify a rule or mapping that associates detailed, intrinsic descriptions of phenomenal characters with detailed, intrinsic descriptions of their corresponding intentional contents.

We are having difficulty finding (or at least agreeing on) a mapping.

\footnotetext{
${ }^{14}$ Harman (1990) also suggests that situation-dependent properties might figure in the contents of visual experiences. See also Schellenberg (2008).
} 
There are two reasons why this might be: it could be that there is no suitable mapping to be found, or it could be that such a mapping exists but is difficult for us to formulate or assess properly. I want to suggest that the second hypothesis should be taken seriously.

We never describe distorted experiences in much detail as far as their intrinsic phenomenal characters go. Instead, we describe the conditions in which they occur. Suppose for example that I want to tell you how a certain squarish object looks to me from my vantage point. All I can say is that the object has such and such squarish shape and that I am viewing it from such and such position relative to it. This does not tell you anything about the perspective-specific phenomenal nature of my experience save for your knowledge that experiences produced in these conditions typically have such and such phenomenal characters. In other words, I cannot articulate the nature of my experience as far as perspective-specific aspects of its phenomenal character go. ${ }^{15}$ Similarly, when we say that an experience is blurry, we

\footnotetext{
${ }^{15}$ One might think that we can say more about the phenomenal characters of our experiences involving perspective by saying how things look in them. For example, when I say that something looks big or small, I might seem to be describing the character of my experience intrinsically. But "look" ascriptions only describe phenomenal characters directly on a phenomenal reading. Jackson (1977) introduced the distinction between phenomenal and non-phenomenal readings of "look." Personally, I cannot hear the phenomenal reading of "look big." When things look big to me, they look to be a size that is big for the relevant kind of thing. For example, for a car to look big to me is for a car to look at least as big as a Cadillac. There is no phenomenal reading of such a claim. As Jackson (1977) acknowledges, the phenomenal reading of "look" is only available when used in conjunction with simple perceptible qualities such as yellowness and squareness. These qualities don't seem to be sufficient to capture the complete phenomenal character of visual experiences involving perspective, because these experiences are not merely experiences of colour arrays. In any case, saying that a cube looks big or small to me would still leave the particular way in which the cube is distorted by perspective unspecified,
} 
are not saying much about its intrinsic nature, only that it is distorted in a certain way compared to normal experiences. Even if blur necessitates a certain kind of phenomenal character (which I doubt), all that I can say about this type of phenomenal character is that it correlates with certain suboptimal perceptual conditions and involves a kind of "blending." I can hardly say anything about the blur-specific aspects of the phenomenal characters of blurry experiences themselves. These and many other examples suggest that it is very difficult if not impossible for us to articulate the phenomenal characters of distorted experiences. For this reason, it looks as if we lack robust concepts for these experiences or their distortion-specific aspects. ${ }^{16}$ I suggest that this explains why we are having difficulty articulating the relationship between contents and phenomenal characters in these cases. The problem is that a clear explanation requires a clear explanandum.

One possible explanation of these shortcomings in our phenomenal concepts is that the properties that are distinctive of the contents of distorted experiences are of very little practical significance. By and large, two kinds of features represented in experience seem to be of practical importance to us. The first are the properties and relations that are instantiated by exeven if such a locution had a phenomenal reading.

${ }^{16}$ By "concept," I mean something more robust than a mere ability to represent a certain feature of phenomenal experience. I mean an ability to think and talk about a certain feature of a phenomenal experience in the same kind of way that we can think and talk about shapes and numbers. It is consistent with what I said above that we have some capacity to introspectively represent all aspects of our phenomenal experiences. What I claim is that this capacity does not enable us to articulate the distortion-specific aspects of our experiences in the kind of way that is required for us to fruitfully theorize about them. 
ternal objects and ourselves, for example, shapes and spatial relations. The second are those properties and relations whose representation in perception is usefully correlated with external features of objects. Arguably, most of the properties traditionally designated as "secondary qualities" fall in this second category. ${ }^{17}$ The properties that undistorted experiences represent seem to include all relevant perceptible features of both of the preceding types. All this leaves for distorted experiences to represent (qua distorted experiences) are exotic features that are not in any way reflected in the outside world. These features are not important to us: they do not help us get around in the world or achieve our aims. Since we have no need to form beliefs or communicate thoughts about these features, it is understandable that we have no concepts or words for them either. If phenomenal character is determined by content as representationalists claim, this could explain why we have so much difficulty articulating the phenomenal characters (and contents) of distorted experiences. ${ }^{18}$ Seen in this light, the present difficulties seem to lend support to representationalism, not to speak against it. ${ }^{19}$

While the preceding account of the ineffability of distorted phenomenal characters is somewhat speculative, the more general point it supports is independently plausible. The point is that the difficulty of articulating how

\footnotetext{
${ }^{17}$ For an extensive discussion of usefully correlated but misrepresented properties, see Mendelovici (2013).

${ }^{18}$ Mendelovici (2014) makes the same point in relation to the contents of emotional experiences.

${ }^{19}$ One might say that it is useful to be able to think such thoughts as my experience is blurry. But recall that "blurry" here does not characterize the phenomenal character of the experience. It is not clear that there is any practical use to talking about blurriness'.
} 
the effects of perceptual distortion on phenomenal characters are reflected in the contents of experience should not be counted as evidence against the view that they are so reflected because it can be traced back to a difficulty in articulating the phenomenal characters of distorted experiences themselves. Any theory of consciousness would struggle to explain these phenomenal characters because they elude theorizing in general. ${ }^{20}$

\section{Conclusion}

My primary aim in this paper was to offer a general answer to a commonly cited class of objections against representationalism, the objections from perceptual distortion. These objections claim that pairs of distorted and undistorted experiences are counterexamples to representationalists' supervenience thesis. My answer is that instances of perceptual distortion that change the phenomenology of an experience also change its content in at least one way: they remove some content that is present in less distorted experiences. This can be made clear by amplifying typical cases of perceptual distortion to a

\footnotetext{
${ }^{20}$ Since normal human vision is arguably always a little blurry and undeniably always involves an element of perspective, what I said in this section suggests that all ordinary visual experiences are ineffable at least in some respect. An anonymous referee suggests that a general skepticism (or perhaps a kind of eliminativism) about experience threatens. Generalized skepticism or eliminativism about experience can be avoided for two reasons. First, it is only some aspects of our experiences that we cannot articulate, not all aspects. Nothing I said suggests that we cannot have beliefs (or knowledge) to the effect that we have experiences. The notion of a phenomenal experience in general is something that we are fully capable of grasping and talking about even if specific aspects of some phenomenal experiences are ineffable. Second, it is consistent with what I said that we have some nonconceptual awareness of all aspects of our experiences, and this is enough to ground a kind of intuitive, inarticulate knowledge of all aspects of our experiences.
} 
point where a great deal of content is lost. This argument works best against putative psychologically possible distortion counterexamples to representationalism, but we saw that it can lend some credibility to the stronger thesis that such counterexamples are not even metaphysically possible.

It is difficult to say exactly how perceptual distortion affects the contents of perceptual experiences. I have suggested that this is due to limitations of our phenomenal concepts: it is not that representationalists' way of explaining phenomenal character is wrong-headed but that the explanandum eludes us conceptually. I have also suggested that these limitations of our phenomenal concepts are due to the contents of distorted experiences involving properties or relations that our conceptual abilities are not tuned to describe. Whether or not this second point is correct, there is no doubt that the phenomenology of perceptually distorted experiences is extremely difficult to articulate. This makes it difficult to articulate how these phenomenal characters correspond to representational contents. For this reason, the difficulties that representational theories face explaining the phenomenal characters of perceptually distorted experiences should not be taken to indicate that these theories are false. Such considerations ought to be borne in mind when assessing the applicability of theories such as representationalism to apparently problematic cases. If the nature of the case is unclear, no theory can be expected to give a clear account of it, because a clear explanation requires a clear explanandum. 


\section{References}

Allen, K. (2013). Blur. Philosophical Studies, 162(2):257-273.

Boghossian, P. A. and Velleman, J. D. (1989). Color as a secondary quality. Mind, 98(January):81-103.

Bourget, D. (2010a). Consciousness is underived intentionality. Nô̂s, $44(1): 32-58$.

Bourget, D. (2010b). The Representational Theory of Consciousness. PhD thesis, Australian National University.

Bourget, D. and Mendelovici, A. (2014). Tracking representationalism. In Philosophy of Mind: The Key Thinkers, pages 209-235. Continuum.

Chalmers, D. J. (2002). Does conceivability entail possibility? In Conceivability and Possibility, pages 145-200. Oxford University Press.

Chalmers, D. J. (2004). The representational character of experience. In The Future for Philosophy, pages 153-181. Oxford University Press.

Dretske, F. (2003). Experience as representation. Philosophical Issues, $13(1): 67-82$.

Harman, G. (1990). The intrinsic quality of experience. Philosophical Perspectives, 4:31-52. 
Jackson, F. (1977). Perception: A Representative Theory. Cambridge University Press.

Jagnow, R. (2009). How representationalism can account for the phenomenal significance of illumination. Phenomenology and the Cognitive Sciences, $8(4): 551-572$.

Kriegel, U. (2011). The Sources of Intentionality. Oxford University Press.

Kuczynski, J.-M. M. (2004). Some arguments against intentionalism. Acta Analytica, 19(32):107-141.

Lycan, W. G. (1996). Consciousness and Experience. MIT Press.

Lycan, W. G. (2000). Representational theories of consciousness. In Stanford Encyclopedia of Philosophy, pages 66-69. Stanford: Metaphysics Research Lab.

Mendelovici, A. (2010). Mental Representation and Closely Conflated Topics. PhD thesis, Princeton University.

Mendelovici, A. (2013). Reliable misrepresentation and tracking theories of mental representation. Philosophical Studies, 165(2):421-443.

Mendelovici, A. (2014). Pure intentionalism about moods and emotions. In Current Controversies in Philosophy of Mind. Routledge.

Millar, B. (2010). Peacocke's trees. Synthese, 174(3):445-461. 
Millar, B. (2013). Colour constancy and fregean representationalism. Philosophical Studies, 164(1):219-231.

Pace, M. (2007). Blurred vision and the transparency of experience. Pacific Philosophical Quarterly, 88(3):328-354.

Peacocke, C. (1983). Sense and Content: Experience, Thought, and Their Relations. Oxford University Press.

Schellenberg, S. (2008). The situation-dependency of perception. Journal of Philosophy, 105(2):55-84.

Schroer, R. (2002). Seeing it all clearly: The real story on blurry vision. American Philosophical Quarterly, 39(3):297-301.

Seager, W. E. and Bourget, D. (2007). Representationalism about consciousness. In The Blackwell Companion to Consciousness. Blackwell.

Smith, A. D. (2008). Translucent experiences. Philosophical Studies, $140(2): 197-212$.

Thompson, B. J. (2006). Color constancy and russellian representationalism. Australasian Journal of Philosophy, 84(1):75-94.

Tye, M. (1996). Perceptual experience is a many-layered thing. Philosophical Issues, 7:117-126. 
Tye, M. (2003). Blurry images, double vision, and other oddities: New problems for representationalism? In Consciousness: New Philosophical Perspectives. Oxford University Press. 